\title{
Density and Mass Distribution of the Spiral Galaxy NGC 3672
}

\author{
Nazzeha A. Daod Mohammed K. Zeki Aziz J. Abdul-Kahader \\ Department of Physics \\ College of Science \\ University of Mosul
}

(Received 25/4/2013 ; Accepted 9/9/2013)

\begin{abstract}
The density and mass of Spiral galaxy NGC 3672 has been studied as a function of the distance from the center of the galaxy. The mass of the three regions of the galaxy (nucleus, disk and halo) have been calculated and compared with other work. The total mass of NGC 3672 is found to be $1.22 \times 10^{11} \mathrm{M}_{\mathrm{o}}$ at a radius of $23.7 \mathrm{kpc}$.
\end{abstract}

Keywords: Spiral galaxies, NGC 3672, galactic density, mass distribution, rotation curve.

\section{NGC 3672 توزيع الكثالة و الكتلة لامجرة الحازونية}

\section{المالهص}

تمت درلسة توزيع الكثافة و الكتلة للمجرة الحلزونية NGC 3672 كدالة للبعد عن مركز المجرة. ت م

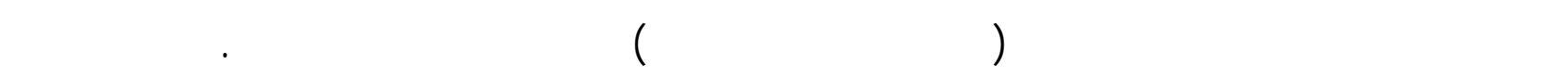

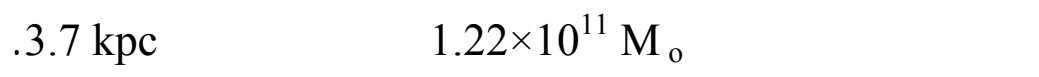

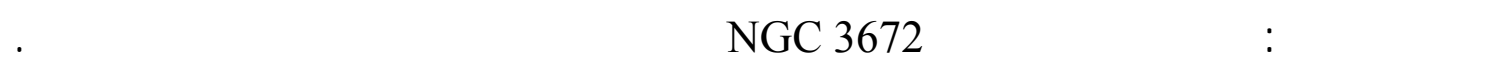

\section{INTRODUCTION}

The determination of galactic mass is an outstanding problem in galactic research. Different methods have been employed leading to widely different results: one method is based upon rotation curves.

Astronomers construct rotation curves for the galaxies from measurements of stars and hydrogen clouds in galactic disks. The rotation curve of a galaxy is the variation in the orbital circular velocity of stars or gas clouds at different radii $r$, (distances from the center of the galaxy). The manner in which the velocity varies with radius reflects the distribution of mass in the galaxy. A great deal of data for the galactic rotation curves have been obtained by various methods such as using the velocities of $\mathrm{H}_{\alpha}, \mathrm{HI}, \mathrm{CO}$, and Maser lines (Sofue, 1996; Deguchi et al., 2000; Maciel and Lago, 2005; Mathewson and Ford, 1996; Greisen et al., 2009).

The rotation of spiral galaxies was discovered in 1914, by Slipher, who detected absorption lines in the nuclear spectra of M31 and Sombrero galaxy. Thereafter, rotation 
curves of galaxies have been constructed by many astronomers (Sofue, 2008; Thakre et al., 2011).

Most spiral galaxies have rotation curves that show solid body rotation in the very center followed by almost a constant (flat) velocity rotation in the outer parts of the galaxy. A flat rotation curve implies that the mass continues to increase linearly with radius, i.e. spiral galaxies contain very massive halos (Sofue and Rubin, 2001). Therefore, the rotation curves of spiral galaxies present a strong evidence of dark matter. Dark matter is a material that does not produce detectable amounts of light but it does have a noticeable gravitational effects. Dark matter is possibly, made of large objects like brown dwarfs, white dwarfs, black holes or small things like neutrinos and other exotic particles that have not been seen in laboratories yet.

In this paper, we studied the galactic density and mass variation with the distance from the center of the galaxy NGC 3672 depending on its observed rotation curve (Sofue et al., 1999).

NGC 3672 (Sc II) is an attractive multi-armed spiral galaxy undistinguished optically except for the small angular extent of its radius (Rubin et al., 1977).

\section{THEORY}

To find the density and mass of a spiral galaxy at an enclosed radius $r$, we started with the following equation (Binney and Tremaine, 1987):

$$
v^{2}(r)=4 \pi G \sqrt{1-k^{2}} \int_{0}^{r} \frac{\rho(a) a^{2} d a}{\sqrt{r^{2}-a^{2} k^{2}}}
$$

where $\rho(a)$ is the galactic density, $k$ is the eccentricity of the galaxy and $v(r)$ is the circular velocity at a distance (r) from the center of the galaxy.

Equation (1) was solved by Daoud et al., (2009) using Abel's integral technique to get a relation for the density law of the form,

$$
\rho(r)=\frac{k^{4}}{\pi^{2} G r \sqrt{1-k^{2}}} \frac{d}{d r} \frac{1}{r} \int_{0}^{r} \frac{v^{2}(a) a d a}{\sqrt{r^{2}-a^{2} k^{2}}}
$$

Again, equation (2) was used by Daod, (2012) to study the density and mass as a function of $\mathrm{r}$ for spiral galaxies which show the general form of rotation curve (steep nuclear rise and high central rotation. followed by a broad maximum in the disk and then a flat rotation due to massive halo), and three different orientations of the flat part were considered (rising up slightly, almost flat and going down slightly).

Daod, (2012) derived the density and the mass of spiral galaxies as a function of the distance from the center of the galaxy. Two sets of equations were derived for the density and the mass, one for the inner parts of the galaxy $\left(r<r_{t}\right)$, where $r_{t}$ is the turn off radius, and the other set of equations are for the outer regions of the galaxy $\left(r>r_{t}\right)$, so

$$
\rho_{1}(r)=\frac{2 \alpha^{2}\left\{2-\sqrt{1-k^{2}}\left(k^{2}+2\right)\right\}}{3 \pi^{2} G \sqrt{1-k^{2}}}
$$

and 


$$
M_{1}(r)=\frac{8 \alpha^{2}\left\{2-\sqrt{1-k^{2}}\left(k^{2}+2\right)\right\}}{9 \pi G} r^{3}
$$

$\rho_{1}(r)$ and $M_{1}(r)$ are the inner density and mass of the galaxy respectively at a given radius $\left(r<r_{t}\right)$.

The density for the outer regions of the galaxy is given by:

$$
\begin{array}{r}
\rho_{2}(r)=\frac{2 \beta^{2}\left\{2-\sqrt{1-k^{2}}\left(k^{2}+2\right)\right\}}{3 \pi^{2} G \sqrt{1-k^{2}}} \\
+\frac{\beta \gamma k\left\{\sin ^{-1} \mathrm{k}-\mathrm{k} \sqrt{1-\mathrm{k}^{2}}\right\}}{G \pi^{2} \sqrt{1-k^{2}} r}
\end{array}
$$

where $\alpha, \beta$, and $\gamma$ are quantities taken from the rotation curve of the given galaxy. Using the following equation:

$$
d M=4 \pi a^{2} \sqrt{1-k^{2}} \rho(a) d a
$$

Then,

$$
M(r)=4 \pi \sqrt{1-k^{2}} \int_{0}^{r} \rho_{2}(a) a^{2} d a
$$

or

$$
\begin{aligned}
M(r)=\frac{8 \beta^{2}\left\{2-\sqrt{1-k^{2}}\left(k^{2}+2\right)\right\}}{9 \pi G} r^{3} & \\
+ & \frac{2 \beta \gamma k\left\{\sin ^{-1} k-k \sqrt{1-k^{2}}\right\}}{G \pi} r^{2}
\end{aligned}
$$

Since the outer mass should be the mass of the outer regions only i.e. the mass within the distance between $r_{t}$ and $r\left(r>r_{t}\right)$. Then

$$
M_{2}(r)=M(r)-M\left(r_{t}\right)
$$

or

$$
\begin{aligned}
M_{2}(r)=\frac{8 \beta^{2}\left\{2-\sqrt{1-k^{2}}\left(k^{2}+2\right)\right\}}{9 \pi G}\left(r^{3}-r_{t}{ }^{3}\right) \\
+\frac{2 \beta \gamma k\left\{\sin ^{-1} k-k \sqrt{1-k^{2}}\right\}}{G \pi}\left(r^{2}-r_{t}{ }^{2}\right)
\end{aligned}
$$

Again $\mathrm{M}_{2}(\mathrm{r})$ is the mass of the outer regions (shell mass) at a distance where $r>r_{t}$. The total mass of the galaxy at any radius $r>r_{t}$ should be

$$
M(r)=M_{1}\left(r_{t}\right)+M_{2}(r)
$$

And the total mass of the galaxy at $\mathrm{r}_{\mathrm{g}}$ (the total radius of the galaxy) should be,

$$
M_{\text {tot }}(r)=M_{1}\left(r_{t}\right)+M_{2}\left(r_{g}\right)
$$




\section{CALCULATIONS AND RESULTS}

Observational data given in Table (1) of the rotation curve of the galaxy NGC 3672 and its plot (Fig. 1) were adopted from Sofue et al.,(1999) to calculate the density and the mass of the galaxy as a function of the distance from its center.

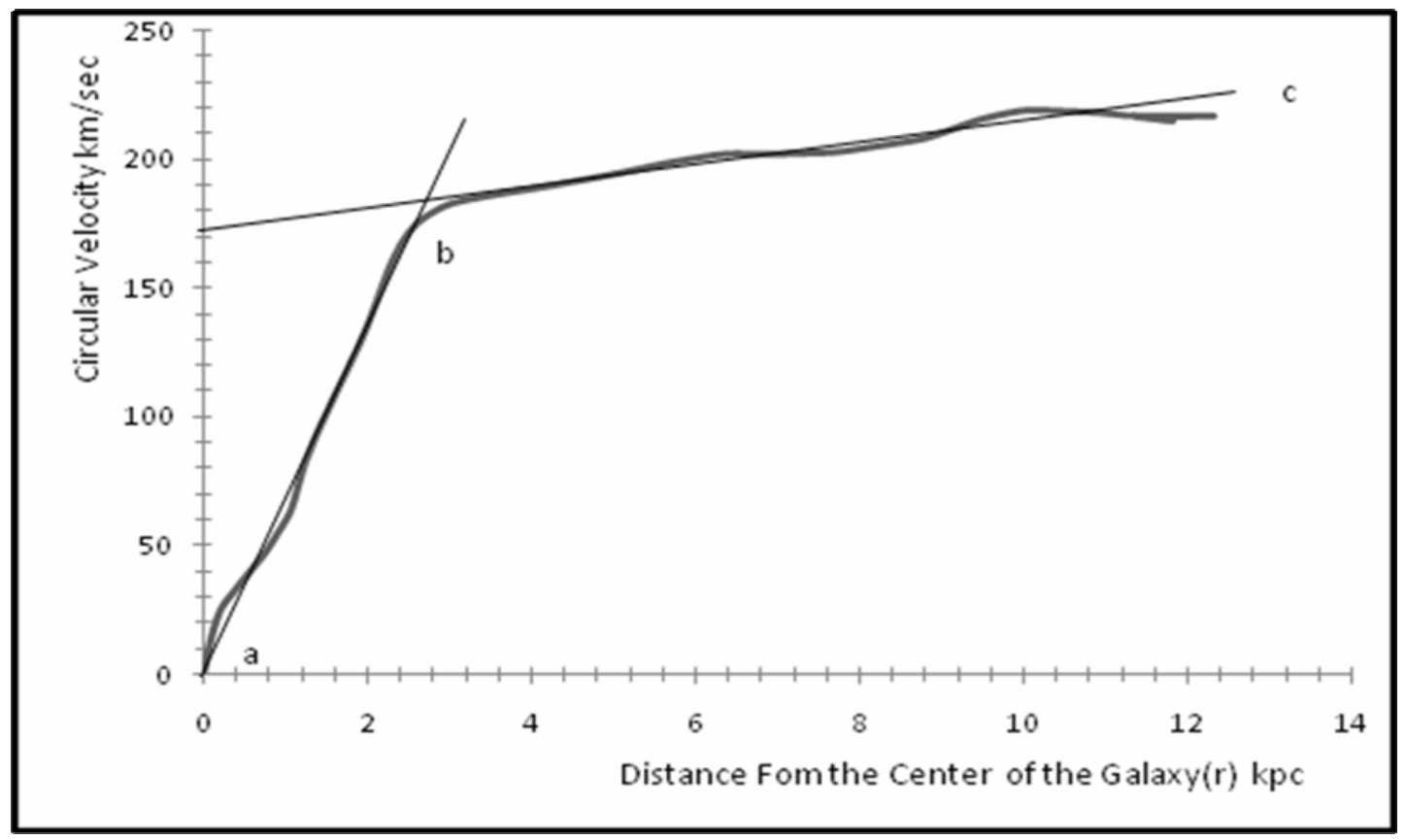

Fig. 1 : Rotation Curve of NGC 3672

Fig. (1) shows the observed rotation curve of NGC 3672 with two fitting lines: the line $a b$ for the radius $r<r_{t}$ and the line $b c$ for the radius $r>r_{t}$. The quantities, $\alpha$ (the slop of the line $a b$ ), $\beta$ (the slop of the line $b c$ ), $r_{t}$ (the turn off radius) and $\gamma$ (the intersection point between the line bc and the vertical axis) were taken from ( Fig. 1) and their values tend to be,

$\alpha=66.67 \mathrm{~km} / \mathrm{sec} / \mathrm{kpc}, \beta=4.35 \mathrm{~km} / \mathrm{sec} / \mathrm{kpc}, \gamma=172 \mathrm{~km} / \mathrm{sec}$ and $\mathrm{r}_{\mathrm{t}}=2.7 \mathrm{kpc}$.

The value of the eccentricity $(k)$ is calculated from $\left(\sqrt{1-k^{2}}=0.1\right)$, the flatness characteristic of spiral galaxies (Binney and Tremaine, 1987).

A computer program in Quick-Basic language was constructed to calculate the density and the mass of NGC 3672 at different distances from the galactic center using the above observational quantities and the equations (3), (4), (5), (10) and (12).

Calculations show that the central (inner) density of NGC 3672 is constant and has a value of $\left(\rho_{1}(r)=1.89 \mathrm{M}_{\mathrm{o}} / \mathrm{pc}^{3}\right)$, where $\mathrm{M}_{\mathrm{o}}$ denotes one solar mass. The density of the outer regions (disk and halo) of the galaxy is much less than that of the inner regions $\left(\rho_{2}(r)<0.09\right.$ $\mathrm{M}_{\mathrm{o}} / \mathrm{pc}^{3}$ ) and it decreases with the distance from the center of the galaxy as shown in (Fig. 2). 


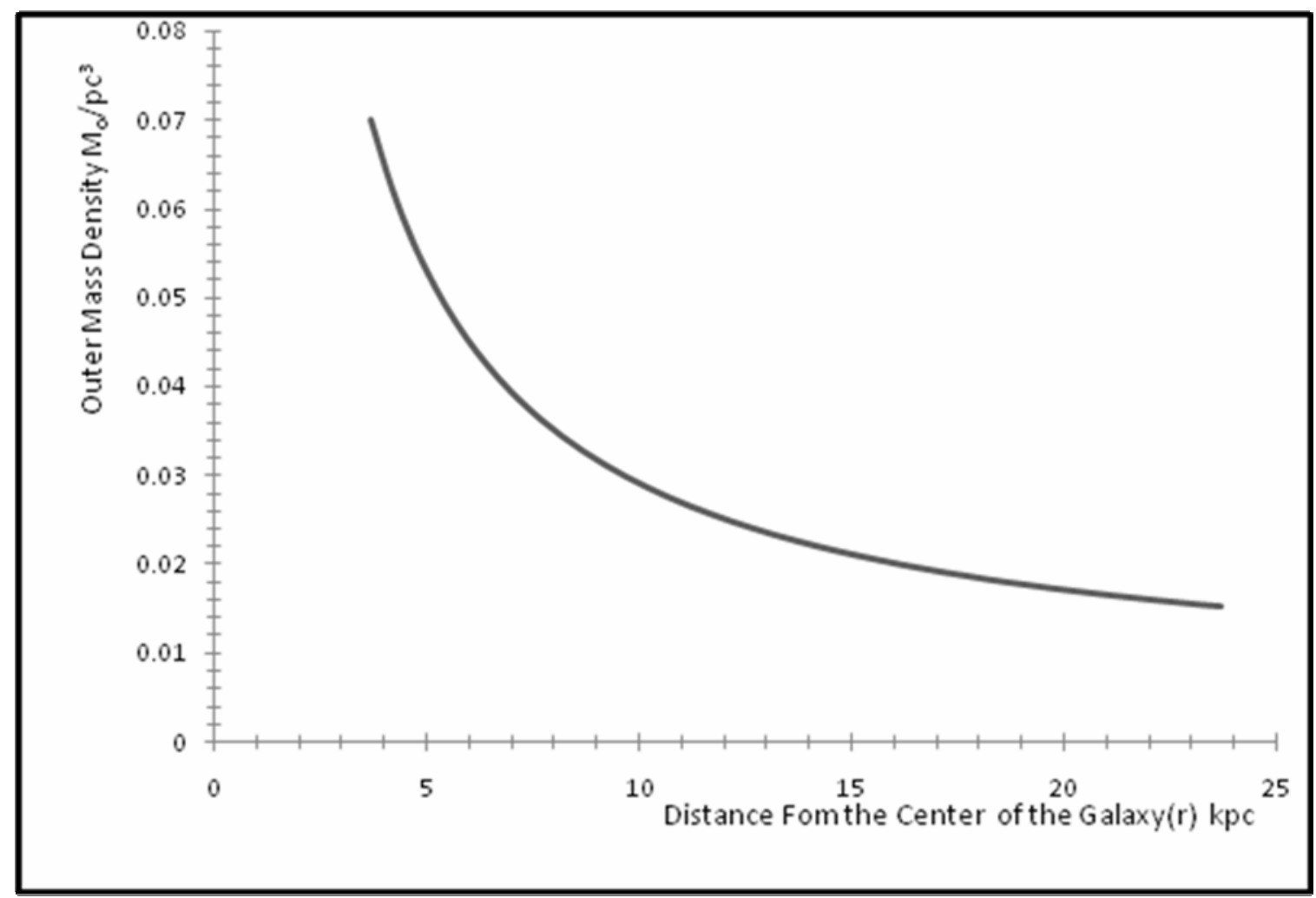

Fig. 2 : The outer Density as a Function of Radius

Our results of constant inner density and declining outer density are consistent with other works (Rubin, et al., 1977 ; Binney and Tremaine, 1987).

The mass of the galaxy NGC 3672 at a radius less than the turn off radius is proportional to $\mathrm{r}^{3}$ (equation 4). Therefore the inner mass of NGC 3672 grows up quikly with distance from the galactic center $(r=0)$ to the turn off radius $\left(r=r_{t}\right)$ reaching a value of $\left(M_{1}\right.$ $\left.\left(\mathrm{r}_{\mathrm{t}}\right)=9.78 \times 10^{9} \mathrm{M}_{\mathrm{o}}\right)$.

The outer mass of the galaxy NGC 3672 at a radius $r>r_{t}$, is found to increase with distance Fig. (3), from the center of the galaxy, although the outer density decreases with radius Fig. (2). All rotation curves for spiral galaxies are approximately flat distances as great as $\mathrm{r}=50 \mathrm{kpc}$ (Rubin et al., 1977). We adopted $\mathrm{r}_{\mathrm{g}}=23.7 \mathrm{kpc}$ from Rubin (1977) to calculate the Halo mass and the total mass of NGC 3672. The halo mass $\left(\mathrm{M}_{\text {halo }}=5.4 \times 10^{10}\right.$ $\left.M_{o}\right)$ is found to be more than the inner mass $\left(M\left(r_{t}\right)=9.78 \times 10^{9} M_{o}\right)$ which confirm the existence of a massive halo and also give a big evidence for the existence of dark matter. Spiral galaxies have giant halos extending to several times the radius of luminous matter (Ostriker et al., 1974). So if the galaxy NGC 3672 extends more than $23.7 \mathrm{kpc}$ then the halo mass will be much more than that calculated above. 


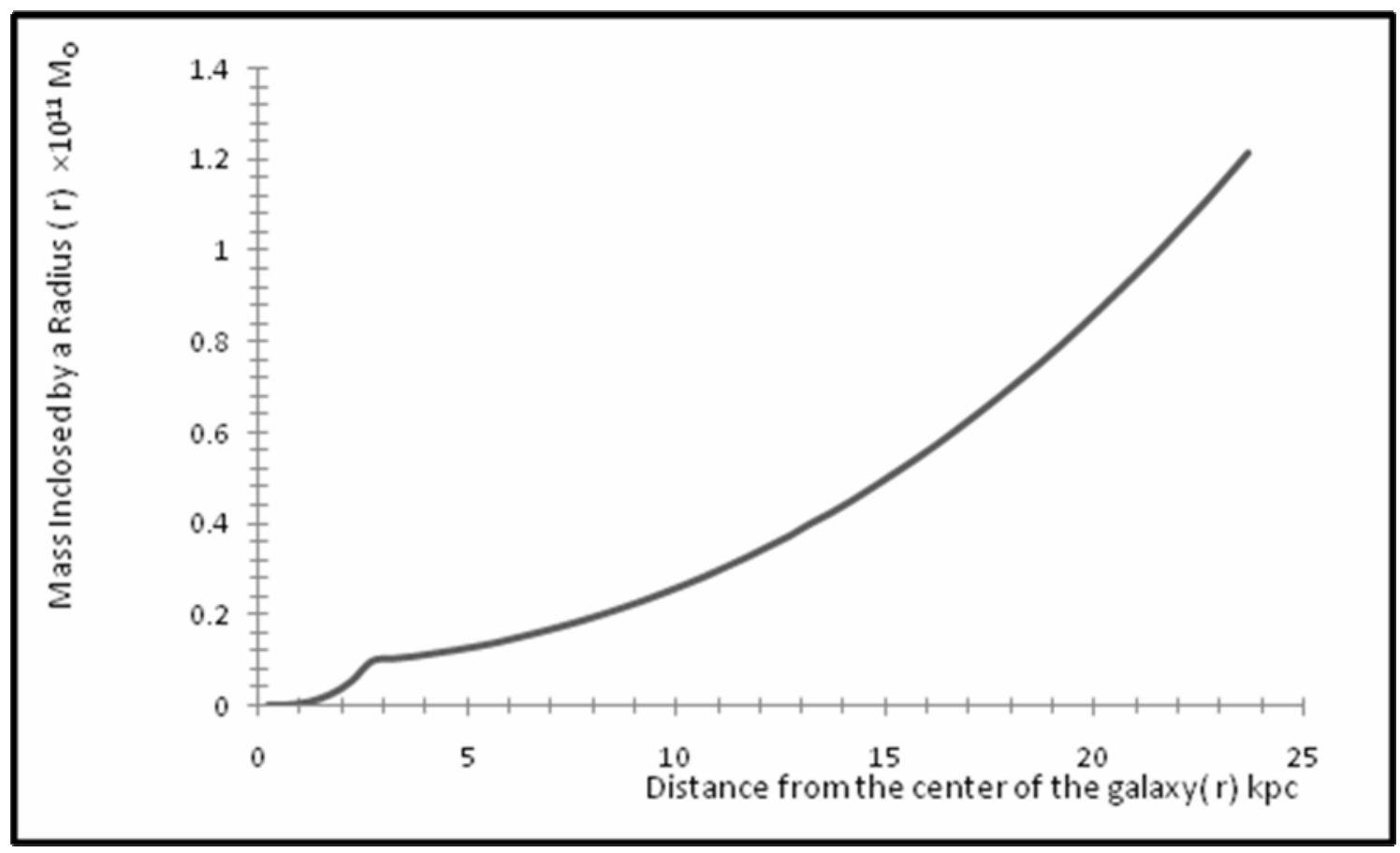

Fig. 3: Mass Distribution of NGC 3672

Spiral galaxies are composed of four components: nucleus, bulge, disk and dark halo (Sofue, 2008). Below the calculated mass of those four components is presented.

The mass of the nucleus of the galaxy NGC 3672 is calculated at a radius of $350 \mathrm{pc}$ and is found to be $\left(2.13 \times 10^{7} \mathrm{M}_{\mathrm{o}}\right)$. The bulge mass is equal to the inner mass at $r=r_{t}$ and is found to be $9.78 \times 10^{9} \mathrm{M}_{0}$. Therefore NGC 3672 has a nucleus of a very small mass compared with the bulges mass.

Emission lines are observed over a distance of $17.6 \mathrm{kpc}$ from the center of NGC 3672. The disk radius is defined as the distance at which luminous objects can be observed, i.e. the disk radius is equal to $17.6 \mathrm{kpc}$ and the calculated mass from equation (10) at this radius turns to be $\left(5.822 \times 10^{10} \mathrm{M}_{\mathrm{o}}\right)$.

The calculated halo mass $\left(5.4 \times 10^{10} \mathrm{M}_{\mathrm{o}}\right)$ at a radius of $23.7 \mathrm{kpc}$ is a large contribution to the total mass of the galaxy $\left(1.22 \times 10^{11} \mathrm{M}_{\mathrm{o}}\right)$ although luminous objects are very rare in the halo. Existence of a massive halo can be explained by the presence of the dark matter in the form of non luminous large objects like brown dwarfs, white dwarfs, neutron stars and black holes or small particles like neutrinos, baryons and unknown elementary particles which have not yet discovered in lab. experiments on earth (non baryonic dark matter).

The total mass of the galaxy NGC $3672\left(1.22 \times 10^{11} \mathrm{M}_{\mathrm{o}}\right)$ at a radius of $23.7 \mathrm{kpc}$ is within the range $\left(1.22 \pm 0.02 \times 10^{11} \mathrm{M}_{\mathrm{o}}\right)$ given by Rubin et al.,(1977). Calculations also show that NGC 3672 has a nucleus of small mass and a massive halo which is consisted with that found by Rubin too.

\section{CONCLUSION}

In this work we calculated the density and mass of the spiral galaxy NGC 3672 at different distances from the center of the galaxy using equations derived by Daod, (2012) and the observed rotation curve from Sofue et al.,(1999). Calculations confirm that the galaxy NGC 3672 has a small nuclear mass and a massive halo. The total mass of the NGC 
3672 at an outer radius of $23.7 \mathrm{kpc}$ is $\left(1.22 \times 10^{11} \mathrm{M}_{\mathrm{o}}\right)$ and this value might grow up if the galaxy extends farther than $23.7 \mathrm{kpc}$.

\section{REFERENCES}

Binney, J.; Tremaine, S. (1987). "Galactic Dynamics ". Princeton University press, USA. Daod, N.A. (2012). The distribution of the density and mass for spiral galaxies. J. Raf. Sei. 23(2), 131-173.

Daoud, N.A.; Abdul-Kahader, A.J.; Zeki, M.K. (2009). Density law of spiral galaxy relative to rotation curves. J. Raf. Sci., 20(3), 90-96.

Deguchi, S.; Fujii, T.; Izumiura, H.; Kameya, O.; Nakada, Y.1. (2000). SIO maser survey of the galactic disk IRAS sources. Ap. J. Suppl., 128, 571.

Greisen, E.W.; Spekkens, K.; Van Moorel, G.A. (2009). Aperture synthesis observations of the nearby spiral NGC 6503: modeling the thin and thick HI disks. Astronomical $J$. 13, $4718-4733$.

Maciel, W.J.; Logo, L.G. (2005). A new determination of the rotation curve from galactic disk planetary nebulae. Revista Mexicana de Astronomiay Astrofisica, 41, 383 388.

Mathewson, D.S.; Ford, V.L. (1996). Parameters of 2447 southern Spiral galaxies for use in Tully-Fisher relation. Ap. J. Suppl. 107, 197.

Ostriker, J.P.; Peebles, P.J.; Yahil, A. (1974). The size and mass of galaxies and the mass of the universe . Astrophysical J. Lett. 193, (L 1 - L4).

Rubin, V.C.; Thonnard, N.; Foed, W.K. (1977). Extended rotation curves of high-luminosity spiral galaxies. I the angle between the rotation axis of the nucleus and the outer disk. Ap. J., 217, (L1-L4).

Sofue, Y. (1996). The most completely sampled rotation curves for galaxies. Ap. J. 458, 120 $-131$.

Sofue, Y. (2008). Pseudo rotation curve connecting the galaxy, dark halo and local group. Astron. Soc. Japan, 1, 77.

Sofue, Y.; Rubin, V. (2001). Rotation curves of spiral galaxies. Annu. Rev. Astron. Astro. Phys., 39, $137-174$.

Sofue, Y.; Tutui, Y.; Honma, M.; Tomita, A.; Takamiya, T.; Koda, J.; Takeda Y. (1999). Rotation curves of spiral galaxies. Astrophysical J., 523, $136-146$.

Thakre, M.; Upadhyaya, G. K.; Thakre, N.; Sharma, R. K.; Vyas, A. (2011). Nuclear steep point amplitude and mass distribution. International J. Physics and Applications, ISSN 0974 - 3103. 3(1), 97 - 103. 
Table 1: Rotation curve of the galaxy NGC 3672

\begin{tabular}{|c|c|c|c|c|c|c|c|c|c|}
\hline R(kpc) & $\mathrm{V}(\mathbf{k m} / \mathbf{s})$ & Rkpc) & $\mathrm{V}(\mathbf{k m} / \mathbf{s})$ & R(kpc) & $\mathrm{V}(\mathbf{k m} / \mathbf{s})$ & R(kpc) & $\mathrm{V}(\mathbf{k m} / \mathbf{s})$ & R(kpc) & $\mathrm{V}(\mathbf{k m} / \mathbf{s})$ \\
\hline 0.000 & 0.00000 & 2.050 & 140.04424 & 4.100 & 189.00208 & 6.150 & 201.15388 & 8.200 & 204.99055 \\
\hline 0.050 & 8.17544 & 2.100 & 144.33340 & 4.150 & 189.27492 & 6.200 & 201.39310 & 8.250 & 205.23523 \\
\hline 0.100 & 15.13671 & 2.150 & 148.70644 & 4.200 & 189.55257 & 6.250 & 201.61047 & 8.300 & 205.47990 \\
\hline 0.150 & 20.56873 & 2.200 & 152.99306 & 4.250 & 189.83353 & 6.300 & 201.79230 & 8.350 & 205.72458 \\
\hline 0.200 & 24.70579 & 2.250 & 157.02589 & 4.300 & 190.11633 & 6.350 & 201.92653 & 8.400 & 205.96925 \\
\hline 0.250 & 27.46013 & 2.300 & 160.69182 & 4.350 & 190.40004 & 6.400 & 202.00821 & 8.450 & 206.21397 \\
\hline 0.300 & 29.48961 & 2.350 & 163.95436 & 4.400 & 190.68407 & 6.450 & 202.04192 & 8.500 & 206.45905 \\
\hline 0.350 & 31.49676 & 2.400 & 166.83565 & 4.450 & 190.96817 & 6.500 & 202.03947 & 8.550 & 206.70548 \\
\hline 0.400 & 33.50391 & 2.450 & 169.37354 & 4.500 & 191.25230 & 6.550 & 202.01483 & 8.600 & 206.95642 \\
\hline 0.450 & 35.49586 & 2.500 & 171.58896 & 4.550 & 191.53644 & 6.600 & 201.97968 & 8.650 & 207.21886 \\
\hline 0.500 & 37.45622 & 2.550 & 173.48656 & 4.600 & 191.82059 & 6.650 & 201.94208 & 8.700 & 207.50446 \\
\hline 0.550 & 39.39904 & 2.600 & 175.08005 & 4.650 & 192.10477 & 6.700 & 201.90767 & 8.750 & 207.82874 \\
\hline 0.600 & 41.34069 & 2.650 & 176.91264 & 4.700 & 192.38916 & 6.750 & 201.88106 & 8.800 & 208.20566 \\
\hline 0.650 & 43.28233 & 2.700 & 177.55305 & 4.750 & 192.67419 & 6.800 & 201.86626 & 8.850 & 208.64209 \\
\hline 0.700 & 45.22398 & 2.750 & 178.57098 & 4.800 & 192.96088 & 6.850 & 201.86575 & 8.900 & 209.13429 \\
\hline 0.750 & 47.27055 & 2.800 & 179.51318 & 4.850 & 193.25090 & 6.900 & 201.87965 & 8.950 & 209.66988 \\
\hline 0.800 & 49.58307 & 2.850 & 180.39392 & .900 & 193.56451 & 6.950 & 201.90561 & .000 & 210.23335 \\
\hline 0.850 & 52.11268 & 2.900 & 181.20168 & 4.950 & 193.84978 & 7.000 & 201.93999 & 9.050 & 210.81155 \\
\hline 0.900 & 5460 & 2050 & 181 & 5000 & 104 & 7. & 201.97919 & 9.100 & 211.39600 \\
\hline 0.950 & 57.28405 & 3.000 & 182.52135 & 5.050 & 194.48186 & 7.100 & 202.02065 & 9.150 & 211.98230 \\
\hline 1.000 & 5 & 3 & 1 & 0 & 0 & 7 & 7 & 0 & 12.5 \\
\hline 1.050 & 62.90426 & 3.100 & 183.44112 & 5.150 & 195.13908 & 7.200 & 202.10555 & 9.250 & 213.15005 \\
\hline 1.100 & 07.00 & 3 & 1 & 0 & 8 & 7 & 202 & 9.300 & 213.72276 \\
\hline 1.150 & 73.09009 & 3.200 & 184.13345 & 5.250 & 195.80585 & 7.300 & 202.19083 & 9.350 & 214.27689 \\
\hline 1.200 & 78.79397 & 3.250 & 184.44948 & 5.300 & 196.14005 & 7.350 & 202.23352 & 9.400 & 214.80081 \\
\hline 1.250 & 3.38112 & 3.300 & 184.75880 & 5.350 & 196.47430 & 7.400 & 202.27647 & 9.450 & 215.28461 \\
\hline 1.300 & 87.38404 & 3.350 & 185.06313 & 5.400 & 196.80855 & 7.450 & 202.32050 & 9.500 & 215.72433 \\
\hline 1.350 & 91.31095 & 3.400 & 18536099 & 4.450 & 197.14258 & 7.500 & 202.36777 & 9.550 & 216.12405 \\
\hline 1.400 & 95.02150 & 3.450 & 185.65012 & 4.500 & 197.47588 & 7.550 & 202.42293 & 9.600 & 216.49379 \\
\hline 1.450 & 98.40882 & 3.500 & 185.92891 & 4.550 & 197.80724 & 7.600 & 202.49358 & 9.650 & 216.84505 \\
\hline 1.500 & 101.78680 & 3.550 & 186.19759 & 5.600 & 198.13437 & 7.650 & 202.58897 & 9.700 & 217.18665 \\
\hline 1.550 & 105.14875 & 3.600 & 186.45798 & 5.650 & 198.45406 & 7.700 & 202.71693 & 9.750 & 217.52263 \\
\hline 1.600 & 108.49166 & 3.650 & 186.71272 & 5.700 & 198.76292 & 7.750 & 202.88029 & 9.800 & 217.85138 \\
\hline 1.650 & 111.81566 & & 186.96431 & & 199.05872 & 7.800 & 203.07555 & 9.850 & 218.16473 \\
\hline 1.700 & 115.12383 & 3.750 & 187.21446 & 5.800 & 199.34131 & 7.850 & 203.29449 & 9.900 & 218.44795 \\
\hline 1.750 & 118.42371 & 3.800 & 187.46419 & 5.850 & 199.61282 & 7.900 & 203.52797 & 9.950 & 218.68243 \\
\hline 1.800 & 121.73177 & 3.850 & 187.71411 & 5.900 & 199.87662 & 7.950 & 203.76863 & 10.00 & 218.85219 \\
\hline 1.850 & 125.08030 & 3.900 & 187.96494 & 5.950 & 200.13597 & 8.000 & 204.01218 & 10.05 & 218.95091 \\
\hline 1.900 & 128.52258 & 3.950 & 188.21771 & 6.000 & 200.39310 & 8.050 & 208.25659 & 10.10 & 218.98521 \\
\hline 1.950 & 132.12804 & 4.000 & 188.47394 & 6.050 & 200.64928 & 8.100 & 204.50122 & 10.15 & 218.97128 \\
\hline 2.000 & 135.96054 & 4.050 & 188.73508 & 6.100 & 200.90376 & 8.150 & 204.74590 & 10.20 & 218.92773 \\
\hline
\end{tabular}

